\title{
APORTES SOBRE NO VIOLENCIA: LA PROPUESTA DEL SOCIALISMO AUTOGESTIONARIO NOVIOLENTO
}

\section{CONTRIBUTIONS OF NON-VIOLENCE: THE PROPOSAL OF NONVIOLENT SELF-MANAGED SOCIALISM}

\author{
Movimiento Humanista \\ elmovimientohumanista@yahoo.com
}

\section{CURRÍCULUM VITAE}

También llamado Nuevo Humanismo, Humanismo Universalista o Movimiento Siloísta, es una corriente de opinión con presencia en más de 100 países de América, Europa, África y Asia, que reconoce los antecedentes históricos del Humanismo y que en la actualidad se entiende más como una nueva sensibilidad, una nueva forma de pensar, sentir y actuar en el mundo. El fundador del Movimiento Humanista es el pensador y escritor argentino Mario Luis Rodríguez Cobos, más conocido por su seudónimo literario Silo. En referencia al seudónimo de este autor, el movimiento a veces es conocido como «movimiento siloísta».

\section{RESUMEN}

Ahora más que nunca existe militarismo y militarización de la sociedad. Pero además, este sistema injusto desarrolla para imponerse y controlar a la población otros muchos mecanismos basados sobre todo en el control de la información, la manipulación de las conciencias, el fomento de la cultura de la evasión y la gestión del miedo y la inseguridad. Creemos que aún no se ha profundizado 
suficientemente en la noviolencia como propuesta global de sociedad, más allá de su dimensión de estrategia de lucha, y esa es nuestra propuesta: construir la sociedad Noviolenta, o Socialismo Autogestionario Noviolento. Se exponen las características de la sociedad noviolenta como autogestión, interculturalidad, superación del patriarcado...

\section{PALABRAS CLAVE}

Militarización - Sociedad - Manipulación - Noviolencia

\section{ABSTRACT}

Now more than ever there are militarism and militarization of society. Furthermore, this system develops unfair to impose and control the population of other mechanisms based primarily on the control of information, manipulation of consciences, promoting a culture of avoidance and management of fear and insecurity. We believe it is not yet deep enough into the overall proposal nonviolence society, regardless of size reduction strategy, and that is our proposal: to build nonviolent society or Nonviolent Self-Governing Socialism. The basic characteristics of nonviolent society as self-management, intercultural, overcoming patriarchy ...

\section{KEY WORDS}

Militarization - Society - Handling - Noviolence 


\section{TEXTO}

Os adjuntamos este aporte difundido por el grupo antimilitarista Tortuga. El documento afirma que "esta sociedad nueva exige un ser humano nuevo; no se podrá dar si no hay personas capaces de vivirla. No se puede imponer por decreto una nueva sociedad, ni la libertad. La persona nueva será libre; superará la inclinación a someterse, a dejarse oprimir, a delegar en personas expertas o salvadoras el protagonismo de la propia vida. Pero la libertad es algo más: una persona libre es solidaria y comunitaria por definición. La libertad encuentra su apoyo y confirmación en la de los demás. Nos alejamos así del concepto de libertad defendido por el liberalismo, que se apoya en el individuo y ve el final de la libertad donde comienza la libertad de los demás".

\section{1.- LA NECESIDAD DE PROPONER UNA ALTERNATIVA SOCIAL}

Ahora más que nunca existe militarismo y militarización de la sociedad: campos de tiro, bases militares, campaña del ejército en las escuelas, gastos militares, industria armamentística, ejércitos profesionales, campaña de lavado de la imagen del ejército con la incorporación de la mujer y las supuestas "misiones humanitarias", OTAN, generalización de conflictos bélicos por intereses económicos e imperialistas... A todo esto le llamamos militarismo en sentido estricto.

Pero además del "instrumento militar", este sistema injusto desarrolla para imponerse y controlar a la población otros muchos mecanismos basados sobre todo en el control de la información, la manipulación de las conciencias, el fomento de la cultura de la evasión y la gestión del miedo y la inseguridad. De un modo especial la creación o "administración" de amenazas y enemigos monstruosos (inmigración, mafias, delincuencia, fundamentalismo islámico, terrorismo internacional...) que 
justifican un mayor control social, el recorte de libertades y el aumento de represión a quien no comparta el pensamiento único del Poder; también el fomento del individualismo y el consumo para anular los deseos de cambio social. Cuando hablamos de todos estos recursos del Poder, estamos hablando de militarismo en sentido amplio.

Ante todo esto creemos que hay que dar una respuesta: todos esos mecanismos de control social que denunciamos, sirven a un sistema social que nos parece injusto, excluyente y destructor, causante de esta terrible sociedad en la que vivimos.

Mientras exista este sistema de organización social mundial existirán los mecanismos de control antes enunciados, ya que a él, y sólo a él, sirven. Por tanto, además de la resistencia y confrontación frente a este Sistema, es hora de ir proponiendo y construyendo una alternativa global.

\section{2.- LA ALTERNATIVA DE LA NOVIOLENCIA.}

Creemos que aún no se ha profundizado suficientemente en la noviolencia como propuesta global de sociedad, más allá de su dimensión de estrategia de lucha, y esa es nuestra propuesta: construir la sociedad Noviolenta, o Socialismo Autogestionario Noviolento.

Escribimos "noviolencia" junto, y desechamos "no violencia" para destacar el carácter activo, provocador y agresivo que tiene para nosotr@s el término. Nada que ver con pasividad, con renuncia a defenderse y defender lo justo. La agresividad es la fuerza de la vida ante las dificultades. La noviolencia es una fuerza respetuosa de la persona y el bien común, al servicio de su desarrollo, y en lucha a muerte con las fuerzas que los destruyen. 
Por "socialismo" entendemos la pasión por la justicia, por garantizar a tod@s el pan y el bienestar (la infraestructura de la vida); pero también esa aspiración a la libertad, al gobierno del ser humano por sí mismo (negación del gobierno de unos seres humanos por otros), una rebelión permanente contra toda opresión, una lucha para poder disponer de la propia vida, la lucha por construir la sociedad como comunidad de personas libres e iguales, como una necesidad de independencia y solidaridad, en la que siempre habrá que seguir luchando por la libertad (F. García).

Después del fracaso del modelo soviético parece una locura proponer un modelo social como alternativa al capitalismo que padecemos, a pesar de sus manifiestas deficiencias: globalización económica, militarización y control social, abismo NorteSur, exclusión, sexismo, resolución violenta de los conflictos, individualismo, tecnologicismo, productivismo, desastre ecológico, consumismo... Los teóricos del Sistema hablan del mejor de los mundos posibles y del fin de la Historia, y son muchas las personas que no lo conciben de otro modo. No obstante estas dificultades creemos que es urgente y necesario construir una alternativa global a este sistema que de otro modo llevaría a la humanidad y al planeta entero directamente al suicidio colectivo.

Hay que ir más allá de la simple oposición y empezar ya a vivir ese otro mundo posible con el que soñamos. Eso sí, hay que aprender de los errores de los intentos anteriores.

No se trata de redactar una macroteoría política, ni de hacer propuestas exhaustivas acerca de cómo se conformaría la sociedad en todos los aspectos. La noviolencia no puede dejar nunca de ser un modelo abierto y complementario con otros. Bastará con esbozar sus líneas maestras u orientaciones, una de las cuales, como no, es que la 
sociedad noviolenta se construye y configura en permanente diálogo con la realidad. Desconfiamos de los caminos únicos, apostamos por consagrar la libertad de experimentación, la innovación, la capacidad creadora, la imaginación... Por su misma naturaleza, ni la libertad ni la sociedad noviolenta se podrán imponer.

Hablamos, pues, de un modelo-proceso abierto y dinámico, sin dogmas que puedan acabar pasando por encima de las personas -como tantas veces-, que jamás puede ni debe ser definido en su totalidad. Casi sería mejor decir que se trata de un marco global de convivencia, dentro del cual caben muchos modelos sociales diferentes. Por todo ello, el trabajo noviolento se dirigirá sobre todo a la transformación de las conciencias.

Nunca insistiremos bastante en esto. Si se pierde esto, se pierde todo.

\section{3.- CARACTERÍSTICAS DE LA SOCIEDAD NOVIOLENTA.}

Algunas de las líneas maestras del Socialismo Autogestionario Noviolento, que proponemos a la luz de experiencias concretas reales:

Autogestión. Descentralización o desconcentración de poder en todos los ámbitos: económico, social, político, cultural... Empoderamiento social, protagonismo de la propia vida, participación en la toma de decisiones que afectan a la propia vida de modo asambleario, organización siempre de abajo a arriba. La sociedad noviolenta será una sociedad de sociedades.

Cultura de la noviolencia. Visión positiva de los conflictos, que siempre existirán. Entendemos conflicto como síntoma de situaciones negativas que hay que resolver, como oportunidad para el crecimiento. Aspiramos a la resolución 
constructiva de los mismos sin que una parte se imponga a la otra. Coherencia entre medios y fines: el fin bueno no justifica cualquier medio. Distinción entre la persona y el papel o rol que representa en la sociedad: atacamos roles y comportamientos perjudiciales, dañinos y destructivos, respetando a la persona que los mantiene. Relaciones cooperativas, de apoyo mutuo, creación de condiciones para el desarrollo personal integral...

Interculturalidad. Autonomía de los pueblos. Internacionalismo solidario. Superación de los conceptos de patria, estado o nación.

Propiedad y utilización colectiva de los medios de producción, comunicación e información.

Superación del patriarcado. Relaciones de género, incluidas las estructurales, basadas en la igualdad y el respeto a la diferencia. Socialización del trabajo doméstico y del cuidado de las personas dependientes.

Modelo de persona noviolenta. Esta sociedad nueva exige un ser humano nuevo; no se podrá dar si no hay personas capaces de vivirla. No se puede imponer por decreto una nueva sociedad, ni la libertad. La persona nueva será libre; superará la inclinación a someterse, a dejarse oprimir, a delegar en personas expertas o salvadoras el protagonismo de la propia vida. Pero la libertad es algo más: una persona libre es solidaria y comunitaria por definición. La libertad encuentra su apoyo y confirmación en la de los demás. Nos alejamos así del concepto de libertad defendido por el liberalismo, que se apoya en el individuo y ve el final de la libertad donde comienza la libertad de $1 @$ demás (F. García). 
Modelo de producción y desarrollo sostenible y ecológico. No todo lo que podemos hacer (técnica) debemos hacerlo (ética). Inclusión de la Naturaleza en el proyecto social. Superación del productivismo y el tecnologicismo primando los valores éticos.

Estilo de vida solidario y ecológico. "Hay que admitir que el Sistema, tal y cómo está concebido y cómo funciona, no admite la eliminación de la pobreza sin más: o se cambia el sistema, o la pobreza seguirá. No existe lo uno sin lo otro. La pobreza no está ahí porque sí, es el Sistema. No se trata solamente de contribuir con una cuota de soci@ de alguna ONG, ni de firmar peticiones. O los habitantes de primer mundo renuncian a su estilo de vida, o no se logrará la justicia ni el bienestar universales. No se puede cambiar el sistema sin renuncias, no existe el cambio social indoloro. $\mathrm{O}$ duele porque se renuncia al estilo de vida inhumano para con los que no lo disfrutan y se abandonan los privilegios, o puede que los excluídos causen dolor si intentan por todos los medios salir de su situación." (A. Velloso)

Por último, es obvio que la Sociedad Noviolenta no es ni puede ser monopolio de ningún grupo aisladamente: es patrimonio de los grupos y personas que sintonizan con sus rasgos a nivel de pensamiento y acción. La construcción del Socialismo Autogestionario Noviolento no puede ser sino sinfónica, fruto de la coordinación de realidades y luchas diversas.

\section{4.- LA REVOLUCIÓN NOVIOLENTA.}

Ese podría ser el nombre del camino o proceso que proponemos para llegar desde esta sociedad que tenemos a la noviolenta. Por ello proponemos el uso del concepto "Revolución Noviolenta": 
Amplia y desarrolla el de "estrategia de la noviolencia activa" (no confundir en cualquier caso la noviolencia activa con interpretaciones pasivas u oportunistas estratégicas- de la noviolencia).

Creemos interesante recuperar la políticamente incorrecta palabra de "revolución", ya que se pretende una transformación radical del orden de cosas actual.

El apellido "noviolenta" habla de una visión integral y diferente de la revolución: no es sólo la transformación de las estructuras económicas, se trata simultáneamente de transformar conciencias y mentalidades (modelo de persona), por lo cual será un proceso revolucionario que no se podrá imponer, lento y largo, nunca cerrado ni acabado. Lo cual no está en contradicción con mantener la confrontación más feroz con el sistema, sus mecanismos e instituciones, y nuestras complicidades.

La puesta en práctica de esta revolución noviolenta tiene dos frentes irrenunciablemente complementarios:

1.-La confrontación: crítica, denuncia, desenmascaramiento, oposición, enfrentamiento, resistencia... Los objetivos aquí son, pues:

La desmilitarización social: frente a todos los mecanismos que desarrolla el sistema para imponerse y mantenerse: militares, policiales, burocráticos, educativos, mediáticos...

Aflorar conflictos: desenmascarar situaciones de dominación encubiertas por la imposición y en ocasiones también por la misma voluntad de sumisión. 
Anticapitalismo: frente a los principios y fundamentos del sistema, sus organismos e instituciones en esta fase de su globalización. Los medios de lucha son los de la estrategia de la noviolencia activa:

No colaboración.

Desobediencia civil.

Acción directa noviolenta.

Educación para la paz.

Contrainformación.

Sabotaje noviolento.

Boicot.

2.-Propuesta y realización práctica de la sociedad noviolenta. Proponemos las líneas maestras de nuestra pretendida sociedad noviolenta al mismo tiempo que el carácter de nuestra revolución. Ello lo hacemos a dos niveles:

a) Nivel teórico. Difusión teórica de nuestro análisis de la realidad y de nuestras propuestas: educación, charlas, contrainformación de todo tipo, talleres, propaganda, participación en foros de opinión, diálogo con otros colectivos y con la sociedad en general... 
b) Nivel práctico. Creación de realidades de tipo político, cultural, social y sobre todo económico en las que se viven los valores y se funciona con las líneas maestras de nuestra pretendida sociedad noviolenta. Son realidades que anuncian y prefiguran a pequeña escala nuestro proyecto global. Es nuestra propuesta de sociedad alternativa que empieza ya surgir y a desarrollarse dentro, paralelamente y a costa de la sociedad que criticamos. Desde ahí vamos tejiendo redes solidarias y vamos creciendo. Aquí toma cuerpo realmente la idea de "liberar espacios".

Los objetivos que trabajamos son:

Transarme: desconcentración de poder y empoderamiento social. Es un proceso de trasvase de poder, protagonismo y recursos desde las élites dominantes y el Estado, hacia la sociedad y las personas.

Liberar espacios: creación de experiencias, dinámicas, ámbitos, en los que se anticipe la Sociedad Noviolenta. Son respuestas autogestionadas y alternativas al sistema, espacios de libertad donde todas las personas puedan desarrollarse plenamente.

Coordinación de realidades en línea con el Socialismo Autogestionario Noviolento y fomento de redes de apoyo mutuo, dibujando así una sociedad emergente.

Y los medios que empleamos:

Creación de realidades alternativas desde la base: cooperativas, iniciativas económicas autogestionadas, redes de consumidores, grupos asamblearios, centros 
sociales alternativos, colectivos de participación política directa, grupos de intercambio personal, ateneos...

Defensa Popular noviolenta como alternativa al modelo militar.

Diálogo con otros colectivos y con la sociedad en general.

Cuidado y armonización de la relación entre las dimensiones personal y política.

En general, medios que procuramos no sean fácilmente recuperables por el sistema.

http://es.groups.yahoo.com/group/el_movimiento_humanista

"... reconciliar no es olvidar ni perdonar, es reconocer todo lo ocurrido y es proponerse salir del círculo del resentimiento. Es pasear la mirada reconociendo los errores en uno y en los otros. Reconciliar en uno mismo es proponerse no pasar por el mismo camino dos veces, sino disponerse a reparar doblemente los daños producidos..." Silo. 